\title{
Evaluation of a new foetal shielding device for pregnant brain tumour patients
}

\author{
Seonghee Kang ${ }^{1,4+}$, Kyeong-Hyeon Kim ${ }^{2+}$, Sang-Won Kang ${ }^{2}$, Dong-Seok Shin ${ }^{2}$, Seungwan Lee ${ }^{3}$ and \\ Jin-Beom Chung ${ }^{1 *}$
}

\begin{abstract}
Background: The present study aimed to propose a new foetal shielding device for pregnant cancer patients to reduce the foetal dose associated with treatment techniques using multiple gantry angles, such as intensity-modulated radiation therapy (IMRT) or volumetric modulated arc therapy (VMAT).

Methods: Three shielding structures were designed to minimise the scattered and leaked radiation from various gantry angles and radiation scattering within the patient. The base-plate part that can be placed on the treatment couch was designed to reduce the scattered and leaked radiation generated at gantry angles located near $180^{\circ}$. A body shielding part that can cover the lower chest and abdomen was designed, and a neck-shielding structure was added to reduce the internal and external radiation scattering from the treatment area. Evaluation plans were generated to assess the foetal dose reduction by the foetal shielding device in terms of the shielding material thickness, distance from the field edge, and shielding component using the flattened $6 \mathrm{MV}$ photon beam (6MV) and flattening filter-free $6 \mathrm{MV}$ photon beam (6MV-FFF). In addition, the effectiveness of the foetal shielding device was evaluated in a pregnant brain tumour patient.
\end{abstract}

Results: The shielding material consisting of three parts was placed on frames composed of four arch shapes with a vertical curved structure, connection bar at the top position, and base plate. Each shielding part resulted in reductions in the radiation dose according to the treatment technique, as the thickness of the shielding material increased and the foetal dose decreased. In addition, a foetal dose reduction of approximately $50 \%$ was confirmed at $50 \mathrm{~cm}$ from the field edge by using the designed shielding device in most delivery techniques. In patients, the newly designed shielding structures can effectively eliminate up to about $49 \%$ of the foetal dose generated from various gantry angles used in VMAT or IMRT.

Conclusions: We designed a foetal shielding device consisting of three parts to effectively reduce the dose delivered to the foetus, and evaluated the device with various treatment techniques for a pregnant patient with brain tumour. The foetal shielding device shielded the scattered/leaked radiation from the treatment machine, and also effectively reduced internal scattering from the treatment area in the patient.

Keywords: Foetal dose, Foetal shielding device, VMAT, Pregnant patient

${ }^{*}$ Correspondence: jbchung1213@gmail.com

†Seonghee Kang and Kyeong-Hyeon Kim have equally contributed to this work

1 Department of Radiation Oncology, Seoul National University Bundang Hospital, 82 Gumi-ro 173 Beon-gil, Bundang-gu, Seongnam-si 13620, Gyeonggi-do, Korea

Full list of author information is available at the end of the article

\section{Background}

Although radiation therapy is rarely performed for pregnant patients, it has been used for pregnant patients with breast, head and neck cancer, Hodgkin's disease, leukaemia, and brain tumour [1-3]. For such patients, it is important to consider target control and saving of the surrounding organs at risk (OARs) as well as foetal dose original author(s) and the source, provide a link to the Creative Commons licence, and indicate if changes were made. The images or other third party material in this article are included in the article's Creative Commons licence, unless indicated otherwise in a credit line to the material. If material is not included in the article's Creative Commons licence and your intended use is not permitted by statutory regulation or exceeds the permitted use, you will need to obtain permission directly from the copyright holder. To view a copy of this licence, visit http://creativecommons.org/licenses/by/4.0/. The Creative Commons Public Domain Dedication waiver (http://creativeco mmons.org/publicdomain/zero/1.0/) applies to the data made available in this article, unless otherwise stated in a credit line to the data. 
reduction. When radiation therapy for a pregnant patient is determined, a suitable treatment strategy and shielding structures to mitigate the potential risk to the foetus by reducing the peripheral dose should be considered $[1,4]$. As recommended by the American Association of Physicists in Medicine Task Group 36 (AAPM TG-36), the dose delivered to the foetus should be maintained below 5 cGy to minimise the adverse biological effects of the radiation, which depend on various factors such as gestational age, equivalent dose, and radiation type [1]. In external beam radiotherapy, the foetal dose can be attributed to radiation leakage from the head of the linear accelerator (LINAC) and scatter from the collimator, blocks, and other objects. To minimise the foetal dose, the distance from the radiation field edge should be kept as far as possible, and an optimum treatment technique with an appropriate field size and beam angles should be used [5-8].

When using techniques with relatively large modulation, such as volumetric modulated arc therapy (VMAT) or intensity-modulated radiation therapy (IMRT), to improve target coverage and normal tissue sparing, the dose delivered to the foetus may be increased by the scattered radiation from various angles. Various treatment strategies such as 3D-conformal radiation therapy (3D-CRT), IMRT, and tomotherapy have been compared with respect to their ability to reduce the foetal dose [912]. David et al. [13] reported that the foetal dose from radiotherapy of glioblastoma during pregnancy can be reduced with IMRT by using a mobile shielding device. Owrangi et al. [8] proposed a custom foetal shield to allow multiple beam angles, and the peripheral dose (PD) measurement was evaluated with and without the shielding structures. However, the previously proposed shielding designs were not sufficient to adequately shield the doses scattered by patients and some delivery techniques, such as VMAT or tomotherapy. In addition, addition or removal of shielding materials such as lead to provide an effective foetal dose shield was difficult.

In patients with Hodgkin's disease, where the distance between the treatment field and the foetus is low, three to five half-value layers (HVLs) of lead should be used to sufficiently shield the dose delivered to the foetus $[1,14]$. However, in patients with brain or head and neck (H\&N) cancer, wherein the distance of the foetus from the field edge would be more than $30 \mathrm{~cm}$, VMAT or IMRT might be considered as a treatment option to improve the therapeutic gain. We designed a new shielding structure for brain and H\&N cancer patients to reduce the dose delivered to the foetus for various treatment techniques using multiple gantry angles. Shielding structures consisting of three parts were designed to effectively reduce the dose delivered to the foetus, and the difference in the foetal dose with or without shielding was evaluated using a modified Rando-phantom to verify the effectiveness of the shielding structures.

\section{Methods \\ Shielding structure design}

The principal sources for the dose delivered to the foetus are (1) treatment head leakage from the LINAC, (2) scattered radiation from the collimators and beam modifiers, and (3) radiation scattered within the patient from the treatment beam. In this study, shielding structures were designed to reduce the doses from these three sources during treatment. In particular, because VMAT can generate scattered or leakage radiation from a continuously rotating gantry head, effective shielding of these sources is essential. Therefore, three shielding structures were designed to minimise the scattered and leaked radiation from various gantry angles and the scattered radiation within the patient. Figure 1 shows schematic diagram of the frames to fabricate the shielding structures. First, the part A for neck shielding was added to reduce the internal and external scattered radiation from the treatment area. The internal scattered radiation generated at the target and directed out of the neck can be shielded with the part A. The part B for body shielding that can cover the lower chest and abdomen was designed. In addition, the part $C$ of base-plate that can be placed on the treatment couch was designed to reduce the scattered and leaked radiation generated at gantry angles located near $180^{\circ}$. The frame was used to prevent the deformation and deflection of the shielding structure fabricated with lead.

\section{Evaluation of the foetal shielding device}

Evaluation plans (VMAT plan with two full arcs and IMRT plan arranged with seven gantry angles) were generated to evaluate the PD reduction by the shielding structures in a rotational delivery technique using a flattened 6-MV photon beam (6 MV) and a flattening filter-free 6-MV photon beam (6 MV FFF), as shown in Table 1 . The prescription dose was $60 \mathrm{~Gy}$, with a daily dose of 2 Gy. All treatment plans were created using the photon optimizer (PO; Varian Medical Systems, Palo Alto, CA) and the anisotropic analytic algorithm (AAA; Varian Medical Systems, Palo Alto, CA). The grid size used for dose calculation was $2.5 \mathrm{~mm}$. Dose deliveries for all plans were performed with TrueBEAMTM using a 2.5-mm high-definition multi-leaf collimator (HD MLC, Varian Medical Systems, Palo Alto, CA). The virtual brain tumour in the modified Rando-phantom was $120 \mathrm{cc}$ (equivalent sphere diameter: $6 \mathrm{~cm}$ ), and at least $95 \%$ of the planning target volume (PTV) was covered with $100 \%$ of the prescription dose. Figure 2 shows the modified Rando-phantom set-up to measure the PD generated 


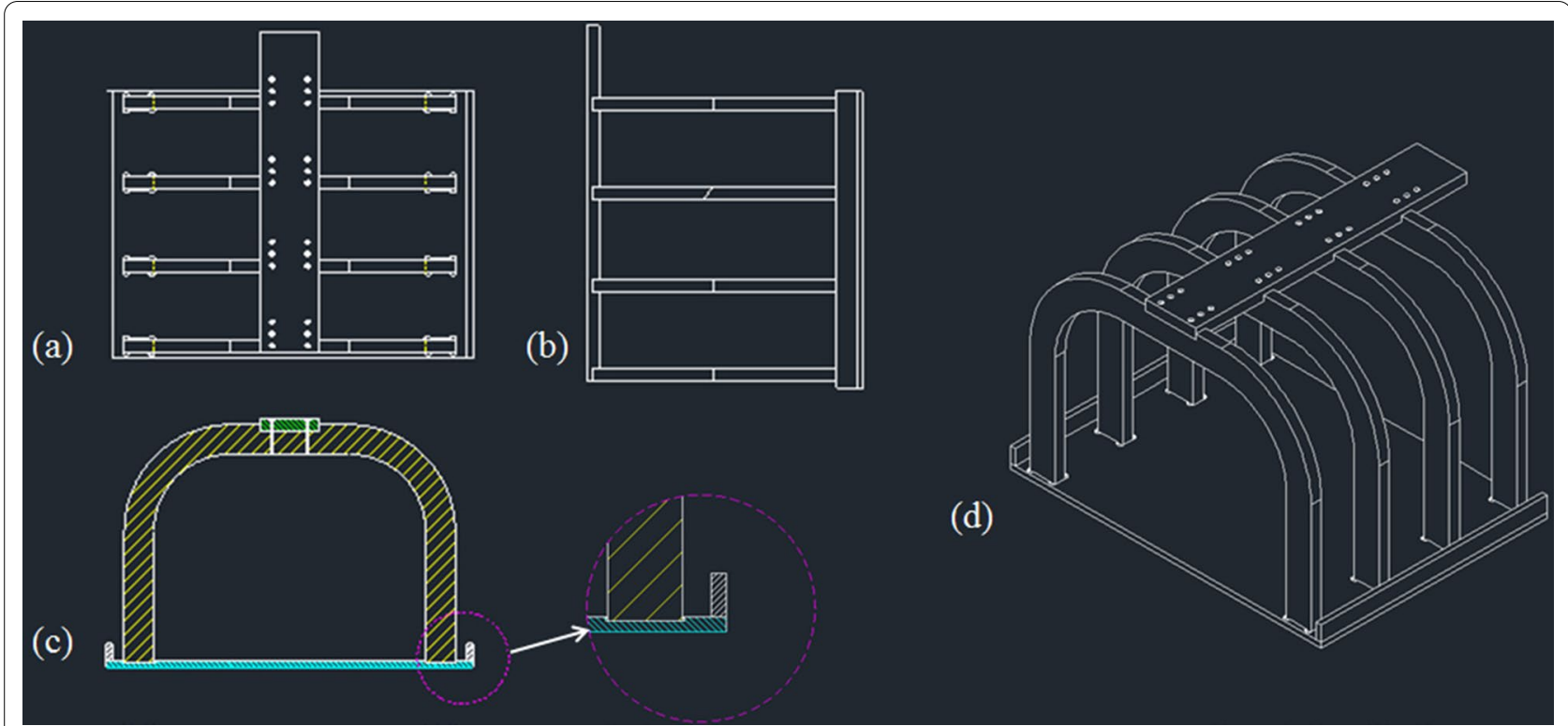

Fig. 1 Schematic diagram of the frame for the shielding material: a top view, $\mathbf{b}$ side view, $\mathbf{c}$ frontal view, and $\mathbf{d}$ the assembled foetal shielding device to support the shielding material

Table 1 Treatment plan for foetal dose evaluation

\begin{tabular}{llll}
\hline Energy & Gantry angle $\left(^{\circ}\right)$ & MU & $\begin{array}{c}\text { Delivery } \\
\text { time (s) }\end{array}$ \\
\hline VMAT & & & 144 \\
6 MV & 2 full arcs & $270 / 265$ & 141 \\
6 MV FFF & 2 full arcs & $272 / 303$ & 185 \\
IMRT & & & 183 \\
6 MV & $50 / 100 / 140 / 180 / 220 / 260 / 310$ & $79 / 89 / 55 / 63 / 52 / 86 / 82$ & $123 / 118 / 79 / 91 / 85 / 111 / 121$ \\
6 MV FFF & $50 / 100 / 140 / 180 / 220 / 260 / 310$ & 183 \\
\hline
\end{tabular}

VMAT volumetric modulated arc therapy, IMRT intensity-modulated radiation therapy, FFF flattening filter-free

during treatment. Since the treatment couch lift capacity should be considered, the appropriate thickness of the shielding material was determined by assessing 6-12$\mathrm{mm}$ lead with a 2-mm thickness interval. In addition, the effectiveness of each component was evaluated by measuring various combinations for each component with VMAT and IMRT plans, and measurements were performed to obtain the doses at a depth of $10 \mathrm{~cm}$ at $30 \mathrm{~cm}$, $40 \mathrm{~cm}$, and $50 \mathrm{~cm}$ distance from field edge to evaluate the dose reduction by the shielding device according to distance. Three repeated measurements were performed using a $0.6-\mathrm{cm}^{3}$ Farmer-type ionisation chamber (Waterproof PTW Farmer Chamber type 30013, Freiburg, Germany), and the measured foetal doses were estimated in terms of average value. The chamber and electrometer were carefully operated to minimise leakage during the measurement.

\section{Patient case}

The patient was 23 weeks pregnant when she was diagnosed with left ventricle glioblastoma (T2 and grade 4) and received postoperative radiotherapy in our department. Computed tomography (CT) simulation was performed to generate a treatment plan with Brilliance CT Big BoreTM (Philips, Cleveland, OH, USA) with a 2-mm slice-thickness. The CT scan range for treatment planning was $36 \mathrm{~cm}$, including head to neck. The patient was immobilised using an Aquaplast facemask (WFR Aquaplast, Wyckoff, NJ). T1 contrast-enhanced and T2 fluid-attenuated inversion recovery (FLAIR) magnetic resonance imaging (MRI) was used to define the target volume, and CT images were imported into the Eclipse treatment planning system (Ver. 13.7, Varian Medical Systems, Palo Alto, CA) for treatment planning. Dose calculation was performed by using 


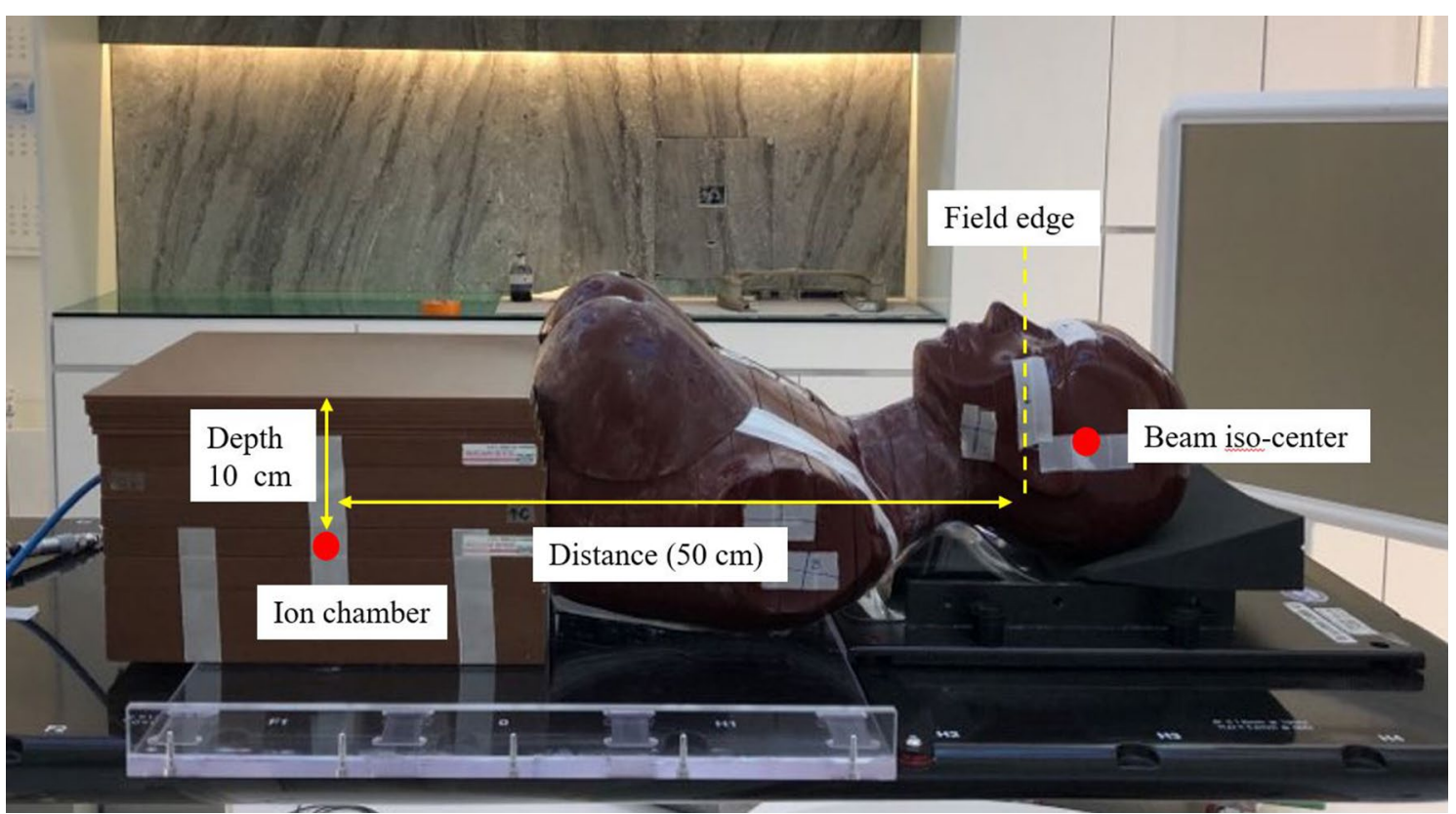

Fig. 2 The modified Rando-phantom set-up for PD measurement. The modified phantom consisted of a Rando-phantom attached to a $30 \times 30 \times 20 \mathrm{~cm}^{3}$ solid water phantom

Table 2 Clinical OAR dose constraints

\begin{tabular}{ll}
\hline Critical structure & Dose constraint \\
\hline Chiasm & Dmax $<55$ Gy to whole structure $(<3 \%)$ \\
Left optic nerve & Dmax $<55$ Gy to whole structure $(<3 \%)$ \\
Right optic nerve & Dmax $<55$ Gy to whole structure $(<3 \%)$ \\
Brainstem & Dmax $<54$ Gy to whole structure $(<5 \%)$ \\
Left eye & Dmax $<15$ Gy to whole structure \\
Right eye & Dmax $<15$ Gy to whole structure \\
Left hippocampus & Dmax $<12$ Gy to $20 \%$ of structure \\
Right hippocampus & Dmax $<12$ Gy to $20 \%$ of structure \\
\hline
\end{tabular}

the analytic anisotropic algorithm (AAA) with heterogeneity correction and a dose calculation gird size of $2.5 \mathrm{~mm}$. A dose of $60 \mathrm{~Gy}$ in 30 fractions was prescribed to the PTV. The primary goal of treatment planning was to cover at least $100 \%$ of the PTV with $95 \%$ of the prescribed dose. Table 2 shows the maximum dose constraint for clinical OARs. A VMAT plan with two partial arcs and an IMRT plan arranged with 7 gantry angles were used to measure the PD reduction by the shielding structures. To maximally separate the treatment site from the foetus position, the treatment couch was not rotated, and the vertex beam, which could deliver the primary beam directly to the foetus, was not used. In addition, for IMRT, the collimator was rotated $90^{\circ}$ to place the distal $\times$ jaws in the superior-inferior direction for the patient. The treatment plans were also generated using the PO and AAA, and TrueBEAMTM using HD MLC was used for beam delivery. Since the distance between the field edge and the umbilicus of the patient is approximately $40 \mathrm{~cm}$, three measurements were performed using a $0.6-\mathrm{cm}^{3}$ farmer-type ionisation chamber at a $10-\mathrm{cm}$ depth with a $40-\mathrm{cm}$ distance from the field edge corresponding to the distance of the umbilicus. In addition, three points have been specified as distances from the field edge $(30 \mathrm{~cm}$ for fundus, $40 \mathrm{~cm}$ for umbilicus, $50 \mathrm{~cm}$ for pubis) according to the TG 36 recommendation, and dose measurements have been performed by using the farmer-type ionisation chamber placed under the $5 \mathrm{~mm}$ bolus at the surface of the phantom. Breasts dose were measured by using the ionisation chamber placed $7 \mathrm{~cm}$ laterally from the central axis and $20 \mathrm{~cm}$ inferiorly from the field edge. The $5 \mathrm{~mm}$ boluses were place under and upper the ionisation chamber.

\section{Results}

Foetal shielding device

As shown in Fig. 3, frames that can support the shielding materials were fabricated. The three parts of the shielding material were placed on frames composed of four arch shapes with a vertical curved structure, a connection bar at the top position, and a base plate. The arch-shaped 


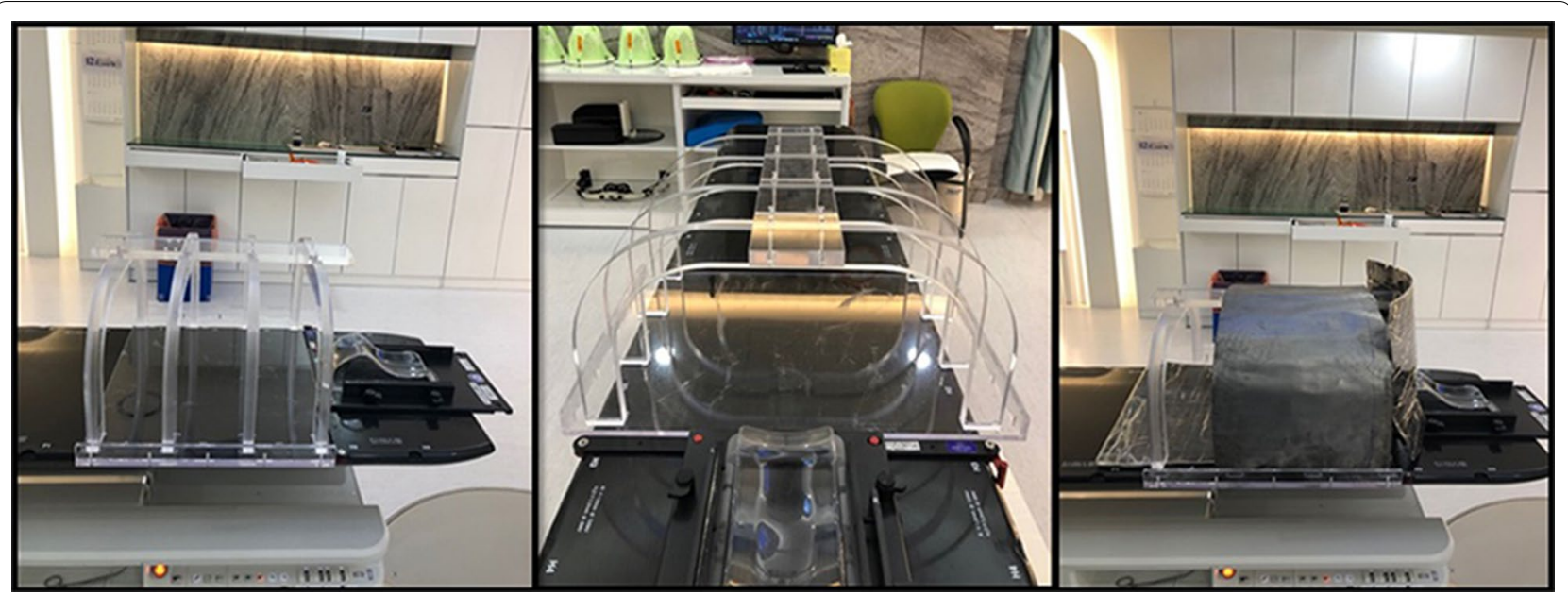

Fig. 3 Photo of the foetal shielding devices with shielding materials (lead)

frame was made of acrylic with a 5-cm thickness to withstand the weight of the shielding materials, and the height and width of the inner cavity were $30 \mathrm{~cm}$ and $46 \mathrm{~cm}$, respectively. The connection bar was designed to change the position of the four arch shaped frames to suit the patient's body shape, and it could support the neck shielding material using the protruding part. The height and width of the inner cavity could be adjusted by using frames of various sizes according to the patient's body size. The base plate was manufactured to be combined with the four arch-shaped frames, and a 2-cm barrier was attached to prevent shielding materials from escaping outside the base plate.

\section{Effectiveness of the foetal shielding device}

Table 3 shows the relative percentage of foetal dose reduction with and without the foetal shielding device to evaluate the shielding effect of each component. Foetal dose measurement was performed $30 \mathrm{~cm}$ away from the field edge using $10-\mathrm{mm}$ lead. In the VMAT technique, all combinations except part $\mathrm{C}$ and parts $\mathrm{A}+\mathrm{B}+\mathrm{C}$ were confirmed to show a greater dose reduction at $6 \mathrm{MV}$ FFF than at $6 \mathrm{MV}$. Among parts A, B, and C, the foetal dose reduction was greater at part $\mathrm{C}$ at $6 \mathrm{MV}(23.58 \%)$ and 6 MV FFF (23.16\%) in the VMAT. When using only two parts, the reduction was greater at $6 \mathrm{MV}$ FFF than at $6 \mathrm{MV}$; on the other hand, when using all parts, the foetal dose decreased more at $6 \mathrm{MV}$. In IMRT, unlike the VMAT, greater reductions were observed at $6 \mathrm{MV}$, except for part A and parts A + C; $6 \mathrm{MV}$ and $6 \mathrm{MV}$ FFF showed greater reductions of $26.41 \%$ and $22.22 \%$ with part $B$ in comparison with parts $\mathrm{A}$ and $\mathrm{C}$. The maximal foetal dose reduction was confirmed at $6 \mathrm{MV}$ with parts $\mathrm{A}+\mathrm{B}$ in comparison with the other two pairs of parts, but greater foetal dose reduction was also achieved by using parts $\mathrm{A}+\mathrm{B}+\mathrm{C}$ at $6 \mathrm{MV}$ than $6 \mathrm{MV}$ FFF.

In order to evaluate the appropriate thickness of shielding materials, the foetal dose reduction with all parts of the foetal shielding device at 2 -mm thickness intervals from 6 to $12 \mathrm{~mm}$ are shown in Table 4. The reduction

Table 3 Relative foetal dose reduction rate with and without each part of $10 \mathrm{~mm}$ shielding device. The point of measurement is from $30 \mathrm{~cm}$ at the field edge and $10 \mathrm{~cm}$ depth from the surface

\begin{tabular}{|c|c|c|c|c|c|c|c|}
\hline & Part A (\%) & Part B (\%) & Part C (\%) & Parts A + B (\%) & Parts A + C (\%) & Parts B + C (\%) & $\begin{array}{l}\text { Parts } \\
\text { A }+ \text { B }+C \\
(\%)\end{array}$ \\
\hline \multicolumn{8}{|l|}{ VMAT } \\
\hline $6 \mathrm{MV}$ & 17.92 & 20.75 & 23.58 & 26.42 & 29.25 & 31.13 & 36.79 \\
\hline $6 \mathrm{MV} \mathrm{FFF}$ & 21.05 & 22.11 & 23.16 & 28.42 & 30.53 & 31.58 & 35.79 \\
\hline \multicolumn{8}{|l|}{ IMRT } \\
\hline $6 \mathrm{MV}$ & 21.50 & 26.41 & 15.37 & 31.31 & 22.73 & 22.73 & 34.99 \\
\hline $6 \mathrm{MV} F F F$ & 22.22 & 22.22 & 13.58 & 25.93 & 25.93 & 20.99 & 33.77 \\
\hline
\end{tabular}

VMAT volumetric modulated arc therapy, IMRT intensity-modulated radiation therapy, FFF flattening filter-free, Part $A$ neck shielding, Part B body shielding, Part $C$ back shielding 
Table 4 Foetal dose reduction at a point $30 \mathrm{~cm}$ away from the field edge for different thicknesses of shielding material with parts $A+B+C$ in comparison with no shielding device

\begin{tabular}{llllll}
\hline & $\begin{array}{l}\text { W/O FSD } \\
\text { (cGy) }\end{array}$ & $\begin{array}{l}\text { 6-mm } \\
\text { lead } \\
\text { (cGy) }\end{array}$ & $\begin{array}{l}\text { 8-mm } \\
\text { lead } \\
\text { (cGy) }\end{array}$ & $\begin{array}{l}\text { 10-mm } \\
\text { lead } \\
\text { (cGy) }\end{array}$ & $\begin{array}{l}\text { 12-mm } \\
\text { lead (cGy) }\end{array}$ \\
\hline $\begin{array}{lllll}\text { VMAT } \\
\text { 6 MV }\end{array}$ & 2.83 & 2.02 & 1.89 & 1.77 & 1.75 \\
6 MV FFF & 2.53 & 1.84 & 1.73 & 1.61 & 1.59 \\
IMRT & & & & & \\
6 MV & 2.19 & 1.57 & 1.44 & 1.41 & 1.36 \\
6 MV FFF & 2.16 & 1.62 & 1.54 & 1.42 & 1.40 \\
\hline
\end{tabular}

VMAT volumetric modulated arc therapy, IMRT intensity-modulated radiation therapy, FFF flattening filter-free, FSD foetal shielding device, W/O without

was greater at $6 \mathrm{MV}$ than at $6 \mathrm{MV}$ FFF for both VMAT and IMRT for all thicknesses. As expected, the foetal dose decreased with an increase in the shielding material thickness. For 12-mm lead, a maximal reduction from 2.83 to 1.75 cGy was observed with 6 MV VMAT, whereas the least reduction from 2.16 to 1.41 cGy was observed with $6 \mathrm{MV}$ FFF IMRT. Interestingly, the difference in the extent of reduction between two successive thicknesses was the smallest $(0.5 \%)$ between 10 and 12-mm lead with the $6 \mathrm{MV}$ FFF IMRT, and the largest (5.9\%) between 6 and $8-\mathrm{mm}$ lead with the 6MV IMRT. The difference between 6 and 6 MV FFF in VMAT was $0.18 \mathrm{cGy}$, showing the largest difference for $6-\mathrm{mm}$ lead, whereas in IMRT, the smallest difference of 0.01 cGy was found with $10-\mathrm{mm}$ lead.

Table 5 shows the foetal doses measured at various distances from the field edge using 10-mm lead with all parts. The dose could be reduced by approximately $50 \%$ at $50 \mathrm{~cm}$ from the field edge by using the foetal shielding device in most delivery techniques. This confirmed that as the distance increased from 30 to $50 \mathrm{~cm}$, the reduction in the foetal dose with the shielding device increased. In comparison with other delivery techniques, when $6 \mathrm{MV}$ VMAT was used, foetal doses measured at $30 \mathrm{~cm}$ and $40 \mathrm{~cm}$ from the edge of the field showed the greatest reduction, and the least reduction was observed in $6 \mathrm{MV}$ FFF IMRT at the $30-\mathrm{cm}$ distance. On the other hand, the greatest dose reduction at $50 \mathrm{~cm}$ from the field edge was achieved with 6 MV FFF VMAT, and the smallest dose reduction at the same distance was achieved with 6 MV IMRT.

\section{Patient case}

Figure 4 shows the dose distributions and dose-volume histograms (DVHs) of various treatment plans that met the clinical objective for target volume and critical structures for brain tumour patients. In comparison with the IMRT plans, the VMAT plans showed better dose distribution and PTV coverage. Table 6 indicates the measured foetal dose at a point approximately $40 \mathrm{~cm}$ away from the field edge at the depth of $10 \mathrm{~cm}$ with and without the foetal shielding device using $10-\mathrm{mm}$ lead. The measurement was carefully carried out by monitoring the leakage dose during each measurement, and dose values were corrected for pressure and temperature. Foetal dose in 6 MV FFF IMRT reduced the most to $48.78 \%$ with a foetal shielding device, whereas the lowest decrease was $44.44 \%$ in the 6 MV FFF VMAT. The highest dose was measured at 6 MV VMAT, and the lowest dose was measured in $6 \mathrm{MV}$ FFF VMAT. Table 7 shows the fundus, umbilicus and symphysis pubis point dose measurement by using the ionisation chamber. All measurement points reduced under 5 cGy with a foetal shielding device, and the dose reductions of the VMAT plans were higher than those of the IMRT plans. The measured breasts dose with and without the foetal shielding device are presented in Table 8. Dose reductions of more than $42 \%$ in the IMRT plans and those of more than $45 \%$ in the VMAT plans were observed.

Table 5 Foetal doses measured at points at various distances from the field edge with and without the foetal shielding device using parts $A+B+C$ with 10-mm lead

\begin{tabular}{|c|c|c|c|c|c|c|}
\hline & $\begin{array}{l}\text { W/O FSD at } 30 \mathrm{~cm} \\
\text { (cGy) }\end{array}$ & $\begin{array}{l}\text { With FSD at } 30 \mathrm{~cm} \\
\text { (cGy) }\end{array}$ & $\begin{array}{l}\text { W/O FSD at } 40 \mathrm{~cm} \\
\text { (cGy) }\end{array}$ & $\begin{array}{l}\text { With FSD at } 40 \mathrm{~cm} \\
\text { (cGy) }\end{array}$ & $\begin{array}{l}\text { W/O FSD } 50 \mathrm{~cm} \\
\text { (cGy) }\end{array}$ & $\begin{array}{l}\text { With FSD at } \\
50 \mathrm{~cm} \text { (cGy) }\end{array}$ \\
\hline \multicolumn{7}{|l|}{ VMAT } \\
\hline $6 \mathrm{MV}$ & 2.83 & 1.77 & 1.39 & 0.87 & 1.17 & 0.59 \\
\hline $6 \mathrm{MV}$ FFF & 2.53 & 1.61 & 1.12 & 0.71 & 0.77 & 0.37 \\
\hline \multicolumn{7}{|l|}{ IMRT } \\
\hline $6 \mathrm{MV}$ & 2.19 & 1.41 & 1.20 & 0.77 & 1.09 & 0.59 \\
\hline $6 \mathrm{MV}$ FFF & 2.16 & 1.42 & 0.99 & 0.64 & 0.80 & 0.48 \\
\hline
\end{tabular}

VMAT volumetric modulated arc therapy, IMRT intensity-modulated radiation therapy, FFF flattening filter-free, FSD foetal shielding device, W/O without 

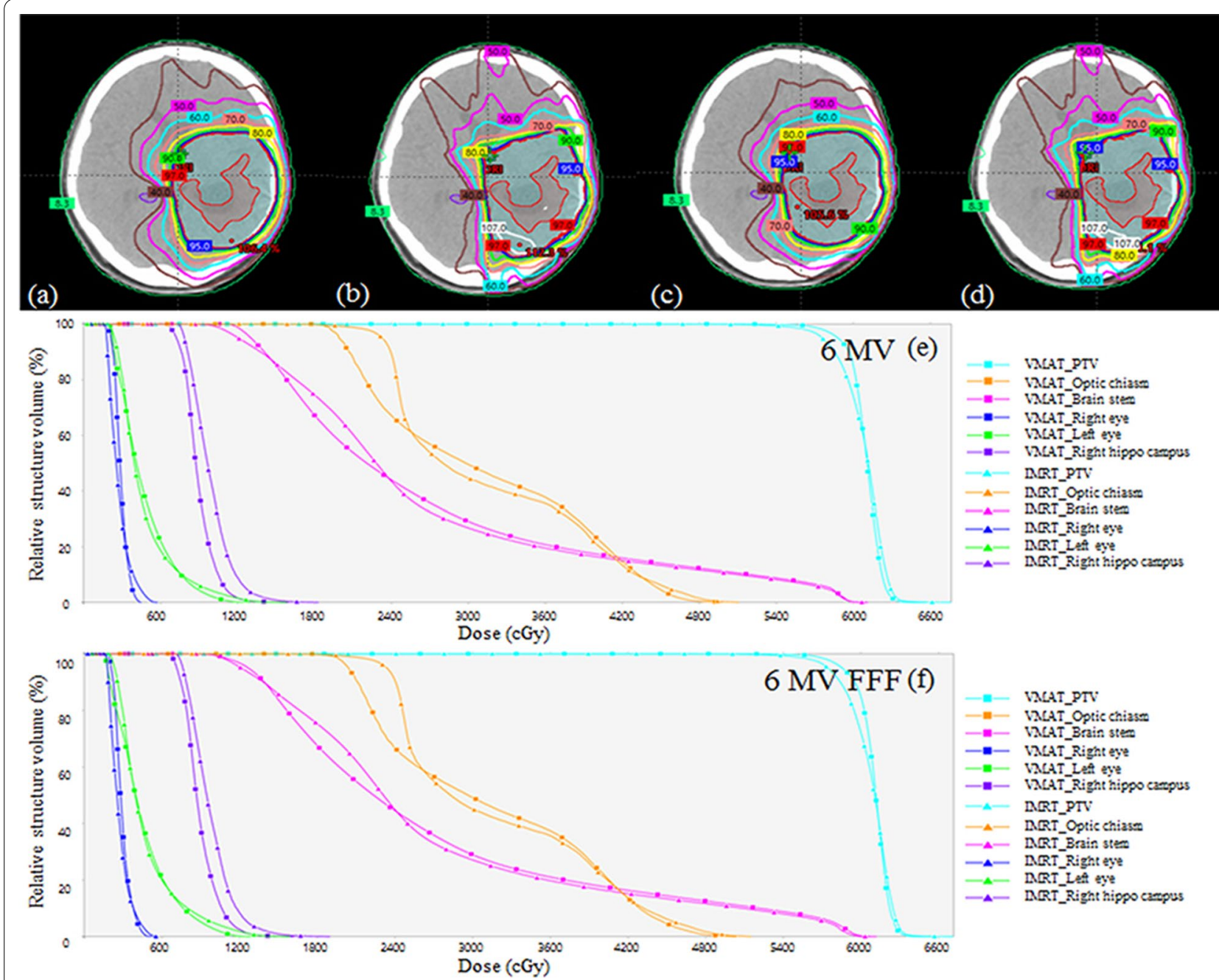

Fig. 4 Dose distributions and DVHs of various treatment techniques for the patient: a 6 MVVMAT, b 6 MV IMRT, c 6 MV FFF VMAT, d 6 MV FFF IMRT, e DVH for 6 MV VMAT and IMRT, and $\mathbf{f}$ DVH for 6 MV FFF VMAT and IMRT

Table 6 The measured foetal doses with and without the foetal shielding device using all parts in a pregnant patient plan at a distance of $40 \mathrm{~cm}$ and $10 \mathrm{~cm}$ depth from surface

\begin{tabular}{llll}
\hline & $\begin{array}{l}\text { FD without FSD } \\
\text { (cGy) }\end{array}$ & $\begin{array}{l}\text { FD with FSD } \\
\text { (cGy) }\end{array}$ & Difference (\%) \\
\hline VMAT & & & \\
6 MV & 3.68 & 2.00 & 45.65 \\
6 MV FFF & 2.88 & 1.60 & 44.44 \\
IMRT & & & \\
6 MV & 3.26 & 1.84 & 45.24 \\
6 MV FFF & 3.28 & 1.68 & 48.78 \\
\hline
\end{tabular}

VMAT volumetric modulated arc therapy, IMRT intensity-modulated radiation therapy, FFF flattening filter-free, FSD foetal shielding device, FD foetal dose

\section{Discussion}

Radiation therapy for pregnant patients should aim to maximise effective and accurate therapeutic effects while minimising the dose delivered to the foetus. Because even small doses delivered to the foetus can be associated with a potentially significant risk, additional care must be taken to confirm to the as low as reasonably achievable (ALARA) principle. According to the AAPM TG Report 36, the risks of radiation to a foetus are classified into seven categories, and adverse biological effects are influenced by various factors such as absorbed dose, type of radiation, and the gestational age at exposure [1]. Once a treatment strategy has been determined, it is necessary to determine whether additional shielding is required and, if necessary, design shielding structures accordingly. 
Table 7 The measured foetal doses with and without the foetal shielding device using all parts in a pregnant patient plan at the three point represented by fundus, umbilicus and symphysis pubis

\begin{tabular}{|c|c|c|c|c|c|c|}
\hline & \multicolumn{2}{|l|}{ Fundus } & \multicolumn{2}{|l|}{ Umblicus } & \multicolumn{2}{|l|}{ Symphysis pubis } \\
\hline & $\begin{array}{l}W / O \text { FSD at } 30 \mathrm{~cm} \\
\text { (cGy) }\end{array}$ & $\begin{array}{l}\text { With FSD at } 30 \mathrm{~cm} \\
\text { (cGy) }\end{array}$ & $\begin{array}{l}\text { W/O FSD at } 40 \mathrm{~cm} \\
\text { (cGy) }\end{array}$ & $\begin{array}{l}\text { With FSD at } 40 \mathrm{~cm} \\
\text { (cGy) }\end{array}$ & $\begin{array}{l}\text { W/O FSD } 50 \mathrm{~cm} \\
\text { (cGy) }\end{array}$ & $\begin{array}{l}\text { With FSD at } \\
50 \mathrm{~cm}(\mathrm{cGy})\end{array}$ \\
\hline \multicolumn{7}{|l|}{ VMAT } \\
\hline $6 \mathrm{MV}$ & 8.882 & 4.764 & 3.876 & 1.938 & 2.019 & 0.969 \\
\hline $6 \mathrm{MV} \mathrm{FFF}$ & 7.267 & 4.037 & 3.149 & 1.534 & 1.534 & 0.727 \\
\hline \multicolumn{7}{|l|}{ IMRT } \\
\hline $6 \mathrm{MV}$ & 7.267 & 4.118 & 4.279 & 2.019 & 3.795 & 2.019 \\
\hline $6 \mathrm{MVFFF}$ & 6.217 & 3.876 & 3.472 & 1.696 & 2.826 & 1.534 \\
\hline
\end{tabular}

VMAT volumetric modulated arc therapy, IMRT intensity-modulated radiation therapy, FFF flattening filter-free, FSD foetal shielding device, FD foetal dose

Table 8 The measured breasts dose with and without the foetal shielding device using all parts in a pregnant patient plan

\begin{tabular}{llllll}
\hline & \multicolumn{2}{l}{ Right breast } & & & \multicolumn{2}{l}{ Left breast } & \\
& W/O FSD & With FSD & & W/O FSD & With FSD \\
\hline VMAT & & & & \\
6 MV & 18.651 & 10.254 & & 19.378 & 10.335 \\
6 MV FFF & 16.148 & 8.801 & & 16.794 & 8.478 \\
IMRT & & & & \\
6 MV & 16.310 & 9.204 & & 15.099 & 8.478 \\
6 MV FFF & 15.179 & 8.720 & & 13.968 & 8.074 \\
\hline
\end{tabular}

VMAT volumetric modulated arc therapy, IMRT intensity-modulated radiation therapy, FFF flattening filter-free, FSD foetal shielding device, FD foetal dose

VMAT usually demonstrates superiority to other delivery techniques in terms of the DVH and dose distribution. However, it is important to thoroughly review the effects of high-modulation techniques such as VMAT or IMRT on foetal dose, since such therapies can increase scattered and leakage doses. The previous shielding structures were mostly designed to shield the upper part of the patient; few shielding structures were proposed to shield the scattered and leakage doses that occur when gantry is located at around $180^{\circ}$ and for the internal scattered radiation $[1,3,4,8]$. Thus, the previously designed structures would not be sufficient for VMAT or IMRT using gantry angles of approximately $180^{\circ}$. In addition, shielding against internal scattering consisting of low-energy Compton-scattered photons should be considered to reduce the peripheral dose. We designed shielding structures composed of three parts to effectively shield the radiation generated from a continuous gantry angle. First, the neck shielding structure (part A) is used to minimise the internal scattered radiation caused by the Compton scattered photon, which is approximately $500 \mathrm{keV}$ [15]. The shielding structure for internal scattering is sufficiently wide to shield the scattered radiation by considering the divergence of the scattered and leakage radiation. Because the shape of the body shielding part made by lead can be changed owing to the characteristics of lead, which is soft and malleable, the four acrylic frames were manufactured to minimise the change in shape. The body frame, which is 5-cm-thick, can support heavy lead and provides enough inner cavities for the patient. Lastly, the base plate was made using acrylic panels, and the patient was set up on the base plate placed on the treatment couch. Using these shielding devices, the gantry head scatter and leakage radiation occurring around all directions can be effectively reduced by fully shielding with lead from all sides of the patient.

By using all parts in IMRT and VMAT techniques at a point $30 \mathrm{~cm}$ from the edge, the foetal dose was reduced by up to about $37 \%$, and when using only two parts, namely, $\mathrm{A}+\mathrm{B}, \mathrm{A}+\mathrm{C}$, and $\mathrm{B}+\mathrm{C}$, the reductions were up to $31.31 \%, 30.53 \%$, and $31.58 \%$, respectively. Thus, omission of one part can result in up to about $12 \%$ greater foetal doses. Since the weight allowance of the Exact IGRT couch (Varian Medical Systems, Palo Alto, CA) was approximately $227 \mathrm{~kg}$, the overall weight of the foetalshielding should be less than $100 \mathrm{~kg}$. The total weight of the baseplate, arch shape frame, connection bar and lead may be approximately $92 \mathrm{~kg}$, which not exceed $100 \mathrm{~kg}$. In accordance with the TG-36 report, a lead thickness of 5 to $7 \mathrm{~cm}$ is sufficient to reduce the PD dose regardless of the energy, but other studies have reported that shielding structures with a smaller thickness were sufficient in a low-energy photon beam $[1,16,17]$. In our study, we used only $6 \mathrm{MV}$ and $6 \mathrm{MV}$ FFF beams, not more than 10 $\mathrm{MV}$, to prevent photo-neutron generation. Therefore, it is necessary to determine the appropriate thickness of the shielding device considering the beam energy, treatment technique, and the weight of the shielding materials. As the thickness of the shielding devices increased, the foetal dose decreased more, but 10-mm lead, which can achieve 
a dose of less than 5 cGy, was used in our study. By using $10-\mathrm{mm}$ lead, it is possible to reduce couch sagging as well as improve the convenience of the patient setup. Owrangi et al. [8] reported that PD decreased as the distance between the treatment site and the measurement point increased. Consistent with their findings, as the distance increased, the foetal dose reduction caused by the foetal shielding device also increased. Particularly, the fundus doses without shielding device were more than 5 cGy in both VMAT and IMRT plans. These are higher than the recommendation of AAPM TG-36, suggesting that the fundus dose should be controlled. However, dose under the 5 cGy were measured at the all point of measurement by using the proposed fetal shielding device. As the pregnancy week increases, the fundus moves upward [1]. Therefore, it would be necessary to increase the shielding thickness depending on the pregnancy week. In the patient case, $6 \mathrm{MV}$ VMAT was performed using the smallest monitor units (MU) and beam delivery time (81 s), but it showed the largest foetal dose. On the other hand, the smallest foetal dose was delivered in $6 \mathrm{MV}$ FFF VMAT, and the beam delivery time was $83 \mathrm{~s}$, similar to the $6 \mathrm{MV}$ VMAT. This is due to the reduction in head leakage caused by the flattening filter, and approximately $20 \%$ reduction in foetal dose in VMAT using 6 MV FFF compared to $6 \mathrm{MV}$ was observed [3]. The average beam delivery time in IMRTs was approximately $230 \mathrm{~s}$, which was increased by approximately 35\% compared to the 6 MV FFF VMAT, and a higher foetal dose was measured. The foetal dose measured in the patient was less than 5 cGy using the proposed foetal shielding device for all treatment techniques. In addition, the breast dose caused by scattered radiation can be reduced by the shielding device. However, it is necessary to assess more patient cases, and further studies on the applicability of these devices to patients with head and neck cancers as well as various brain tumours are required.

\section{Conclusions}

We created a three-part foetal shielding device to effectively reduce the dose delivered to the foetus and evaluated a variety of treatment techniques for a pregnant patient with brain tumour. The newly developed shielding devices were able to effectively eliminate scattered/ leakage radiation generated at various gantry angles used in VMAT or IMRT. This shielding device can be easily adapted to the patient to minimise the peripheral dose in many other situations such as CT simulation, imaging dose for patient setup, and radiation therapy for young patients. Using the $6 \mathrm{MV}$ FFF VMAT technique, better dose distribution and shorter delivery time were confirmed, and when applied with the shielding structures, the lowest foetal dose could be delivered. This approach may help reduce adverse effects by minimising the doses delivered to the foetus while effectively treating pregnant patients with the latest treatment techniques.

\begin{abstract}
Abbreviations
OARs: Organs at risk; AAPM TG-36: American Association of Physicists in Medicine Task Group 36; LINAC: Linear accelerator; IMRT: Intensity-modulated radiation therapy; VMAT: Volumetric modulated arc therapy; 3D-CRT: 3D-conformal radiation therapy; PD: Peripheral dose; HVLs: Half-value layers; H\&N: Head and neck; $6 \mathrm{MV}$ : $6 \mathrm{MV}$ photon beam; $6 \mathrm{MV}$-FFF: Flattening filter-free $6 \mathrm{MV}$ photon beam; PO: Photon optimizer; AAA: Anisotropic analytic algorithm; HD MLC: High-definition multi-leaf collimator; PTV: Planning target volume; CT: Computed tomography; FLAIR: Fluid-attenuated inversion recovery; MRI: Magnetic resonance imaging; DVHs: Dose-volume histograms; ALARA: As low as reasonably achievable; MU: Monitor units.
\end{abstract}

\section{Acknowledgements}

Not applicable.

\section{Authors' contributions}

$J B C$ supervised the project, had given valuable advices on the proceeding of this work, and designed the concept and the experiment method of the research. SK and SL supported on data collection and the characterization of the sample. KHK, SWK and DSS performed the experiment and data analysis. SK and KHK wrote the paper and prepared Figures and Tables. JBC revised the manuscript. All authors read and approved the final manuscript.

\section{Funding}

This work was supported by the National Research Foundation of Korea (NRF) Grants funded by the Korea Government (MSIT) (Nos. 2020R1C1C100936611 and 2018R1D1A1B07049159).

\section{Availability of data and materials \\ The datasets supporting the study conclusions are included within this manuscript.}

\section{Declarations}

\section{Ethics approval and consent to participate}

All procedures performed in studies involving a human participants was in accordance with the ethical standards of the institutional research committee. For this type of study, formal consent is not required.

\section{Consent for publication}

Not applicable.

\section{Competing interests}

S Kang, KH Kim, SW Kang, DS Shin, S Lee, JB Chung declare that they have no competing interests.

\section{Author details}

'Department of Radiation Oncology, Seoul National University Bundang Hospital, 82 Gumi-ro 173 Beon-gil, Bundang-gu, Seongnam-si 13620, Gyeonggi-do, Korea. ${ }^{2}$ Department of Biomedical Engineering, Research Institute of Biomedical Engineering, College of Medicine, The Catholic University of Korea, Seoul, Korea. ${ }^{3}$ Department of Radiological Science, Konyang University, Daejeon, Korea. ${ }^{4}$ Present Address: Department of Radiation Oncology, Seoul National University Hospital, Seoul, Republic of Korea.

Received: 11 January 2021 Accepted: 10 June 2021

Published online: 13 June 2021

\section{References}

1. Stovall M, Blackwell CR, Cundiff J, Novack DH, Palta JR, Wagner LK, et al. Fetal dose from radiotherapy with photon beams: report of AAPM Radiation Therapy Committee Task Group No. 36. Med Phys. 1995;22(1):63-82. 
2. Mazonakis M, Varveris H, Fasoulaki M, Damilakis J. Radiotherapy of Hodgkin's disease in early pregnancy: embryo dose measurements. Radiother Oncol. 2003;66(3):333-9.

3. Labby ZE, Barraclough B, Bayliss RA, Besemer AE, Dunkerley DA, Howard SP. Radiation treatment planning and delivery strategies for a pregnant brain tumor patient. J Appl Clin Med Phys. 2018;19(5):368-74.

4. Pregnancy and Medical Radiation. ICRP Publication 84. Ann. 2000;30(1).

5. Mutic S, Esthappan J, Klein EE. Peripheral dose distributions for a linear accelerator equipped with a secondary multileaf collimator and universal wedge. J Appl Clin Med Phys. 2002;3(4):302-9.

6. Fraass BA, van de Geijn J. Peripheral dose from megavolt beams. Med Phys. 1983;10(6):809-18.

7. Van der Giessen PH. A simple and generally applicable method to estimate the peripheral dose in radiation teletherapy with high energy $x$-rays or gamma radiation. Int J Radiat Oncol Biol Phys. 1996;35(5):1059-68.

8. Owrangi AM, Roberts DA, Covington EL, Hayman JA, Masi KM, Lee C, et al. Revisiting fetal dose during radiation therapy: evaluating treatment techniques and a custom shield. J Appl Clin Med Phys. 2016;17(5):34-46.

9. Zhang P, Happersett L, Hunt M, Jackson A, Zelefsky M, Mageras G. Volumetric modulated arc therapy: planning and evaluation for prostate cancer cases. Int J Radiat Oncol Biol Phys. 2010;76(5):1456-62.

10. Studenski MT, Bar-Ad V, Siglin J, Cognetti D, Curry J, Tuluc M, et al. Clinical experience transitioning from IMRT to VMAT for head and neck cancer. Med Dosim. 2013;38(2):171-5

11. Ezzell GA, Burmeister JW, Dogan N, LoSasso TJ, Mechalakos JG, Mihailidis D, et al. IMRT commissioning: multiple institution planning and dosimetry comparisons, a report from AAPM Task Group 119. Med Phys. 2009;36(11):5359-73.

12. Pasler M, Wirtz H, Lutterbach J. Impact of gantry rotation time on plan quality and dosimetric verification-volumetric modulated arc therapy (VMAT) vs. intensity modulated radiotherapy (IMRT). Strahlenther Onkol. 2011;187(12):812-9.

13. Horowitz DP, Wang TJ, Wuu CS, Feng W, Drassinower D, Lasala A, et al. Fetal radiation monitoring and dose minimization during intensity modulated radiation therapy for glioblastoma in pregnancy. J NeuroOncol. 2014;120(2):405-9.

14. Woo SY, Fuller LM, Cundiff JH, Bondy ML, Hagemeister FB, McLaughlin P, et al. Radiotherapy during pregnancy for clinical stages IA-IIA Hodgkin's disease. Int J Radiat Oncol Biol Phys. 1992;23(2):407-12.

15. Chofor N, Harder D, Willborn KC, Poppe B. Internal scatter, the unavoidable major component of the peripheral dose in photon-beam radiotherapy. Phys Med Biol. 2012;57(6):1733.

16. Magne N, Marcie S, Pignol J, Casagrande F, Lagrange J. Radiotherapy for a solitary brain metastasis during pregnancy: a method for reducing fetal dose. Brit J Radiol. 2001;74(883):638-41.

17. Ngu SC, Duval $P$, Collins $C$. Foetal radiation dose in radiotherapy for breast cancer. Australas Radiol. 1992;36(4):321-2.

\section{Publisher's Note}

Springer Nature remains neutral with regard to jurisdictional claims in published maps and institutional affiliations.
Ready to submit your research? Choose BMC and benefit from:

- fast, convenient online submission

- thorough peer review by experienced researchers in your field

- rapid publication on acceptance

- support for research data, including large and complex data types

- gold Open Access which fosters wider collaboration and increased citations

- maximum visibility for your research: over 100M website views per year

At BMC, research is always in progress.

Learn more biomedcentral.com/submissions 